\title{
Un modelo teórico-práctico: la transpodidáctica textual. Usos y aplicaciones de los textos de ficción para la enseñanza y aprendizaje de lenguas
}

\author{
Pilar COUTO CANTERO \\ Departamento de Didácticas Específicas \\ Universidad de La Coruña. UDC. España. \\ pilar.couto@udc.es
}

Recibido: diciembre 2012

Aceptado: junio 2014

\section{RESUMEN}

El objetivo principal de este artículo consiste en la presentación de un modelo teóricopráctico que hemos denominado: "modelo de la transpodidáctica textual". Este modelo está basado en la elaboración y aplicación de una metodología desarrollada en cuatro fases con la finalidad de demostrar los beneficios resultantes de la implementación de la misma en la enseñanza y aprendizaje de lenguas en contextos educativos. Para demostrar la validez de este modelo se ha utilizado un texto de ficción literario como ejemplo, el cuento de Andersen: "Los trajes nuevos del emperador". Partiendo, por tanto, de ese texto de ficción literario inicial se explicarán los pasos del proceso transpodidáctico textual que ha tenido lugar hasta convertirse en otro texto de ficción diferente, que ha adquirido los rasgos de otro género literario, en este caso una obra teatral breve, y que ha sido elaborado con la finalidad de ser utilizado para la enseñanza y aprendizaje de lenguas.

Palabras clave: Transpodidáctica textual, aprendizaje de lenguas, educación.

\section{An Action-Research Model for the Teaching and Learning Languages Process}

\begin{abstract}
The main aim of this article is to present a new educational theoretical model based in the elaboration and put into practice of a new methodology developed in order to demonstrate the benefits of using fictional texts with educational purposes in the learning languages domain. The transformation from "The emperor's new clothes" fairy tale written by Andersen into a drama play elaborated by teachers and performed by foreign languages learners has been used as an example. This in four steps based model has been implemented for Primary School level as an example, but it could also be satisfactorily implemented in Early Childhood, Secondary and Adult Education levels with many interesting outcomes.
\end{abstract}

Keywords: Fictional Texts, Action-Research, Teaching, Languages, Education. 


\section{Un modèle théorico-pratique: la transpodidactique textuelle. Utilisation et applications pour l'enseignement et l'apprentissage des langues}

\section{RÉSUMÉ}

L'objet principal de cet article est la présentation d'un modèle théorico-pratique que nous avons nommé: modèle de la transpodidactique textuelle. Ce modèle se base sur l'élaboration et l'application d'une méthodologie développée en quatre phases avec la finalité de montrer les avantages de l'implémentation de celle-ci dans l'enseignement et l'apprentissage de langues dans des contextes éducatifs. Pour démontrer la validité de ce modèle on a proposé un texte de fiction littéraire comme exemple, le conte d'Andersen: $L a$ nouvelle robe de l'empereur. À partir, donc, de ce texte de fiction littéraire initiale, on va expliquer les phases du processus transpodidactique textuelle qui fera que ce texte devienne un autre texte de fiction différent, qui aura acquis les traits d'un autre genre littéraire, dans ce cas une œuvre théâtrale brève, et qui aura été élaboré dans le but d'être utilisé pour l'enseignement et l'apprentissage de langues.

Mots clé: transpodidactique textuelle, apprentissage des langues, éducation.

SUMARIO: 1. Introducción 2. Modelo teórico-práctico: la transpodidáctica textual 2.1. Estructura del modelo transpodidáctico textual 2.2 Validación del modelo transpodidáctico textual 3. Propuesta concreta: "Los trajes nuevos del emperador" 3.1 Selección y elección del texto de ficción literario inicial (TFL 1) 3.2 Proceso transpodidáctico textual (Q) 3.3. Texto de ficción literario final (TFT 2) 3.4 Puesta en escena y representación teatral (R) 4. Conclusión 5. Bibliografía

\section{INTRODUCCIÓN}

Para iniciar la argumentación del modelo teórico-práctico que presentamos en este trabajo comenzaremos por exponer algunas definiciones que delimitan el significado de lo que entendemos por un modelo teórico $\mathrm{y}$, en concreto, la definición de lo que es una teoría educativa. De acuerdo con Kerlinger (1975) una teoría es "un conjunto de construcciones hipotéticas, unas definiciones y proposiciones relacionadas entre sí, que ofrecen un punto de vista sistemático de los fenómenos, al especificar las relaciones existentes entre variables, con objeto de explicar y predecir los fenómenos". Un año más tarde, Black y Champion (1976) matizan la definición afirmando que una teoría constituye un conjunto de proposiciones que están sistemáticamente relacionadas entre sí y que especifican relaciones causales entre variables. Por último, Moore (1980) define una teoría como un instrumento para la explicación y predicción razonado. Atendiendo a cualquiera de estas tres definiciones acerca del significado del término en cuestión intentaremos demostrar que el objeto de estudio planteado en el título de este artículo encaja perfectamente con la definición de las mismas.

No obstante, aunque atendiendo a estas definiciones, conviene precisar que en este artículo nos referimos, más que a una teoría científica a un modelo teórico educativo en el sentido de que la finalidad de la misma consiste en proporcionar 
una guía para dirigir las prácticas docentes, entendiendo que éste puede ser prescriptivo y que nos recomendará qué tenemos que hacer con respecto a esas prácticas. Un modelo educativo pretende también orientar las prácticas reales del profesorado en las aulas de aprendizaje y justifica además el uso de ciertos medios educativos sobre los que nos centraremos más adelante. Al mismo tiempo, establece un puente entre la práctica educativa y la investigación, proporcionando nuevos modos de enfrentarse a los problemas educativos desarrollado herramientas útiles que permiten encontrar soluciones a dichos problemas y, de algún modo, servirán para predecir que van a funcionar en situaciones nuevas. Las teorías educativas descansan sobre una serie de supuestos como el de uniformidad de la naturaleza y el de causalidad. Además deberán proporcionar información acerca de los objetivos que se persiguen como un fin deseable, los medios a utilizar, la naturaleza del conocimiento y la de los sujetos que deben ser educados y la forma en que se desarrollan y aprenden los seres humanos.

Para acotar el espacio de esta propuesta hemos preferido utilizar el término modelo teórico-práctico en lugar de proponer una teoría que sería un término mucho más ambicioso. Por tanto, para exponer nuestro modelo teórico-práctico sobre la transpodidáctica textual y sus posibles usos y aplicaciones para la enseñanza aprendizaje de lenguas en contextos educativos hemos partido de la teoría de la transposición cinematográfica desarrollada hace tiempo en el campo de la semiótica fílmica y con motivo de otras investigaciones previas enmarcadas en áreas interdisciplinares (Couto Cantero, 1999). En ese estudio se planteaban y analizaban, entre otras cosas, los cambios que se producían durante el proceso de transformación de un texto de ficción literario, por ejemplo una novela, en un texto de ficción fílmico, por ejemplo un filme. Conviene también poner de manifiesto, desde el punto de vista de la didáctica, que los enormes avances en el estudio y uso de las nuevas tecnologías de la comunicación en todos los ámbitos de la sociedad actual, que han sido implantados en todos los curricula nacionales como una de las ocho competencia básicas que todo ciudadano debe de adquirir a lo largo de su formación, han supuesto un elemento importante a tener en cuenta para la realización de esta propuesta.

Por último, la unión de los conceptos: "transposición cinematográfica", que se relaciona claramente con las nuevas tecnologías de la información y de la comunicación y su posterior implementación y aplicación en contextos educativos en el ámbito de la enseñanza reglada, por una parte, y el nacimiento del área de conocimiento denominada: "didáctica de la lengua y la literatura" por otra, han dado lugar a la creación y propuesta de este modelo educativo desde una nueva perspectiva interdisciplinar. El uso y utilidad de los modelos teóricos llevados a la práctica real en las aulas hace que esta investigación adquiera un sentido práctico y global favoreciendo, por tanto, la necesidad de este estudio en el que se describe, explica y valida la propuesta del modelo teórico-práctico de la transpodidáctica 
textual aplicada a la enseñanza y aprendizaje de lenguas para una sociedad plurilingüe y multicultural.

El término transposición didáctica que delimita por primera vez el universo transposicional dentro del campo de la pedagogía no es nuevo. Aunque existe un consenso considerable al atribuir la paternidad del mismo a Michel Verret en el año 1975, fue Yves Chevallard quien por primera vez acuñó el término en el ámbito de la didáctica de las matemáticas en el año 1985 para referirse al proceso de transformación que se produce al convertir un objeto de saber en un objeto de enseñanza. Chevallard distinguió la transposición didáctica en un sentido muy amplio que podría ser representada por el esquema: objeto de saber, objeto a enseñar y objeto de enseñanza, de la transposición didáctica en un sentido mucho más estricto entendiendo ésta como el paso de un contenido de saber preciso a una versión didáctica de este objeto del saber:

... un contenido del saber teórico que haya sido designado como saber a enseñar sufre a partir de entonces un conjunto de transformaciones adaptativas que van a hacerlo apto para formar parte de los objetos de enseñanza. El 'trabajo' que un objeto de saber a enseñar hace para transformarlo en un objeto de enseñanza se llama transposición didáctica (Chevallard, 1985, 39).

Para aclarar mejor este concepto, pongamos por ejemplo el caso de un docente con amplios conocimientos en cuanto a contenidos relativos a diferentes áreas afines. El proceso de transformación y todos aquellos cambios que surgen a partir de la implementación de esos conocimientos en el área objeto de estudio con una finalidad claramente pedagógica podría denominarse, aunque con ciertas reservas, una transposición didáctica. Por lo tanto, desde el punto de vista de la aplicación teórica que proponemos, no parece suficiente el mero hecho de estar en posesión de una gran cantidad de conocimientos y contenidos, sino que el verdadero reto consistirá en saber adaptarlos a los niveles educativos correspondientes y ser capaces de transmitirlos correctamente para que sean aprehendidos por el mayor número de discentes. En definitiva, y de acuerdo con las indicaciones pautadas a través del Marco Común Europeo de Referencia para las Lenguas, desde el punto de vista del profesorado, no solo es necesario adquirir conocimientos o saberes sino que son también muy importantes los conceptos de saber hacer, saber enseñar, saber aprender, saber vivir y saber devenir (Díaz Corralejo, 2002).

\section{MODELO TEÓRICO-PRÁCTICO: LA TRANSPODIDÁCTICA TEXTUAL}

Una vez aclarada la terminología y su procedencia es necesario indicar que, puesto que la teoría de la transposición didáctica ya existe y ha sido utilizada en un contexto diferente al que hoy pretendemos explorar, hemos creído conveniente 
añadir un desriptor más preciso que sirva para representar y definir mejor la propuesta que proyectamos a continuación. Por ello, proponemos la inclusión de esta nueva terminología acuñada por primera vez en esta investigación y a la que hemos denominado: el modelo teórico-práctico de la transpodidáctica textual.

El elemento novedoso de este modelo de la transpodidáctica textual consiste en la implementación de un proceso de transposición de distintos textos de ficción que da lugar a la posterior elaboración de otros textos ficcionales con fines educativos. Concretamente y para este artículo hemos basado la explicación en el ámbito de la enseñanza y aprendizaje de lenguas. Conviene también aclarar que, por cuestiones de diferente índole sobre las que no procede extenderse en este espacio limitado, el ejemplo seleccionado para explicar el proceso y fases de esta propuesta se centra en la aplicación del modelo de la transpodidáctica textual para fundamentar la adquisición y aprendizaje de una lengua extranjera. Trataremos de probar cómo a lo largo de todo el proceso de aplicación desde el inicio hasta el final es posible cumplir ese fin deseable. El seguimiento de esta propuesta se materializa en un ejemplo concreto y en la elección de una lengua concreta, pero únicamente constituye una muestra y/o punto de partida para posteriores análisis de otros procesos transpodidácticos y lenguas en los que se desee implementar el modelo. Otro de los objetivos pretendidos consiste en facilitar a la comunidad educativa, que así lo desee, una sistematización o modus operandi que sirva de guía para futuras actuaciones en aspectos relacionados con el tema objeto de estudio.

\subsection{Estructura del modelo transpodidáctico textual}

Partiendo de las premisas de Moore (1980) acerca de qué es y cómo se estructura una teoría de aplicación práctica entendemos que la estructura de este modelo transpodidáctico textual se puede articular del siguiente modo. En primer lugar, planteamos una hipótesis: el aprendizaje y uso de una lengua extranjera (en adelante LE) constituye un fin deseable, luego $R$ es un fin deseable. Nuestra deducción a partir de esta hipótesis es la siguiente: dado un texto de ficción literario inicial (P1), el proceso transpodidáctico textual (Q) es la mejor forma de conseguir un texto de ficción literario final (P2) con la finalidad de alcanzar el aprendizaje y buen uso de una LE (R). Luego, dadas ciertas circunstancias previas (P1), Q es la mejor forma de conseguir P2 para obtener R. Para comprobar y reforzar esta evidencia empírica, también se podría concluir que nunca se ha encontrado que Q no sea la mejor forma de conseguir P. 


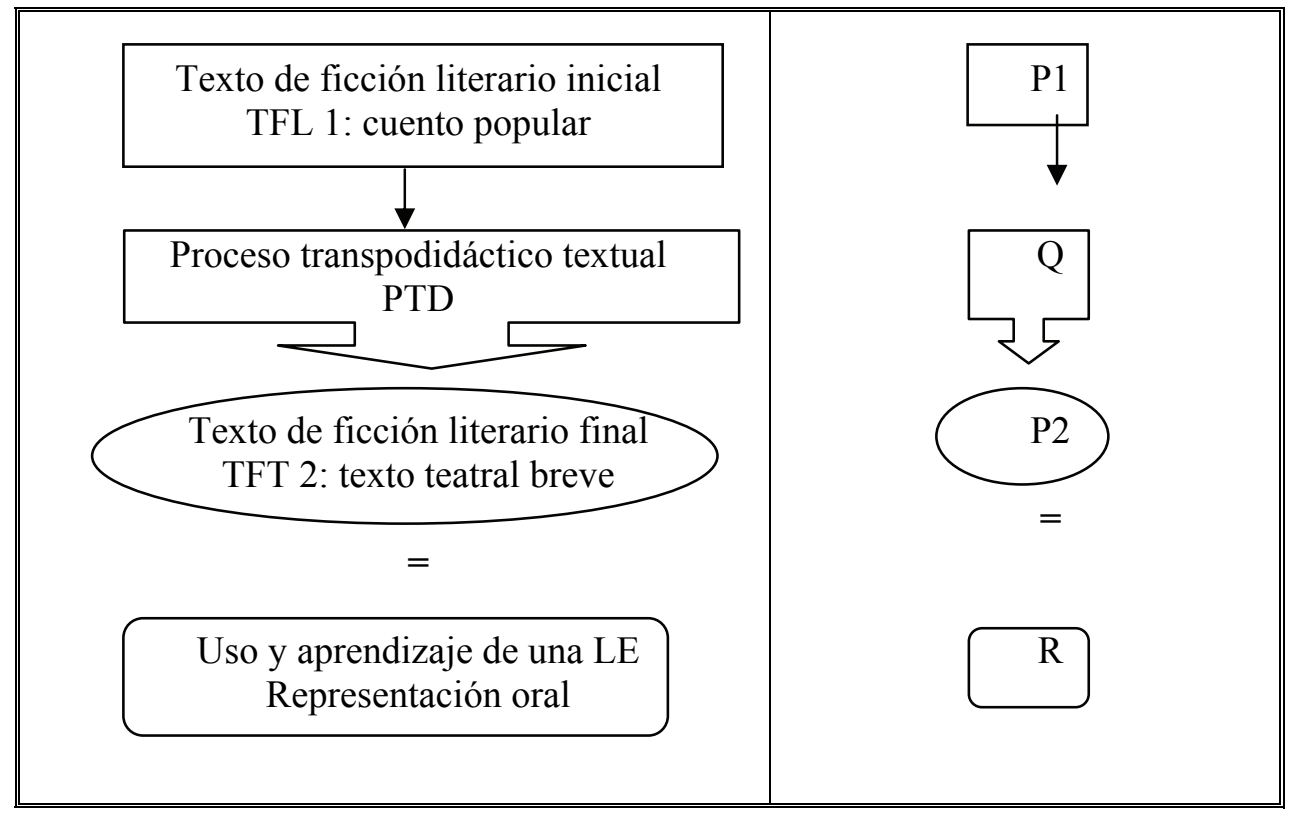

Figura 1. Cuadro resumen del modelo transpodidáctico textual.

Desde el punto de vista de la argumentación filosófica se puede afirmar que los fines que se persiguen en un modelo de este tipo son moralmente válidos, cumpliendo además el requisito de la coherencia interna puesto que existe una clara relación entre los supuestos planteados y los medios empleados para obtenerlos. Luego la conclusión final podría ser que sería conveniente o que habría que implementar Q.

Partiendo de un texto de ficción literario inicial (P1) y por medio de una serie de pasos que serán acometidos a lo largo de un proceso transpodidáctico textual (Q) obtenemos otro texto de ficción literario final totalmente distinto del primero (P2) que presenta un objetivo claramente deseable que es el uso y aprendizaje de una lengua (R). Para ser más concretos, el texto de ficción literario (en adelante TFL) que hemos elegido para ejemplificar esta teoría es un cuento de Hans Christian Andersen: "Keiserens nye Klæder" (Los trajes nuevos del emperador/The Emperor's New Clothes) publicado por primera vez en danés en el año 1837 y que hace tiempo que goza de un sitio preferente dentro de los cánones de la Literatura Infantil Universal.

Por medio de la aplicación del modelo transpodidáctico textual veremos cómo este texto inicial (TFL 1) ha sido transformado y se ha convertido en otro texto de ficción teatral breve (en adelante TFT) que, sin dejar de tener su referente hipotextual en el cuento antes indicado, ha dado lugar a un nuevo producto que tiene una finalidad claramente formativa (TFT 2). Para mayor abundamiento, este 
nuevo texto convertido en una obra teatral breve adaptada al nivel y contexto educativo correspondientes ha de ser representado al final del proceso como evidencia palpable de la consecución de los objetivos inicialmente planteados (R).

Si bien este ejemplo de un TFL convertido en un TFT sirve para dotar de coherencia a nuestro modelo teórico-práctico y constituye el hilo conductor del mismo, parece oportuno aclarar antes de continuar, que existen otras muchas combinaciones posibles para disponer los diferentes textos de ficción a los que nos hemos referido y que éstas se pueden ejecutar en cualquier lengua de aprendizaje. Se puede partir, por tanto, de un TFL y convertirlo en otro TFL, o en un TFT, o también en un texto de ficción fílmico (en adelante TFF); o partiendo de un TFT convertirlo en un TFL, o en un TFF, o en otro TFT y así en sucesivas combinaciones. Ver cuadro a continuación:

\begin{tabular}{|c|c|c|c|c|}
\hline P 1 & & $\overline{\bar{Q} \mathbf{Q}}$ & $\overline{P \text { P 2 }}$ & $\bar{~} \overline{\mathbf{R}}$ \\
\hline $\begin{array}{l}\text { TFL } 1 \\
\text { Texto de ficción literario }\end{array}$ & $\rightarrow$ & $\mathrm{Q}$ & $\longleftrightarrow \begin{array}{l}\text { TFL 2 } \\
\text { TFT 2 } \\
\text { TFF 2 }\end{array}$ & $=\mathrm{R}$ \\
\hline $\begin{array}{l}\text { TFT } 1 \\
\text { Texto de ficción teatral }\end{array}$ & $\rightarrow$ & Q & $\longleftrightarrow \begin{array}{l}\text { TFL 2 } \\
\text { TFT 2 } \\
\text { TFF 2 }\end{array}$ & $=R$ \\
\hline $\begin{array}{l}\text { TFF } 1 \\
\text { Texto de ficción fílmico }\end{array}$ & $\rightarrow$ & Q & $\longleftrightarrow \begin{array}{l}\text { TFL 2 } \\
\text { TFT 2 } \\
\text { TFF 2 }\end{array}$ & $=R$ \\
\hline
\end{tabular}

Figura 2. Otras posibles combinaciones transpodidáctico-textuales.

\subsection{Validación del modelo transpodidáctico textual}

Los elementos que validan los modelos científicos se fundamentan en la capacidad de descripción, explicación y predicción de los mismos. La descripción se refiere a la capacidad para definir el fenómeno, sus características y componentes, así como las condiciones bajo las que se presenta. La explicación se refiere a la capacidad de establecer las causas del fenómeno y proporcionar pruebas empíricas de las mismas. Por último, la predicción se centra en función de la evidencia empírica proporcionada, que si conseguimos que sea constante, se presupone que así será en el futuro. Por tanto, y de acuerdo con las premisas de Hernández y cols. (1998) este modelo transpodidáctico textual que presentamos 
está dotado de consistencia lógica puesto que las proposiciones que lo integran están interrelacionadas y no presentan incoherencias ni contradicciones internas.

Además está dotado también de perspectiva y es a la vez fructífero porque ofrece la posibilidad de generar nuevas preguntas y descubrimientos, cumpliendo además el requisito denominado parsimonia dada su simplicidad y sencillez. Otros rasgos que dotan de validez a este modelo son, por una parte, el rasgo de la universalidad que hace posible que se pueda poner en práctica en cualquier otro contexto cultural distinto de éste en el que nos encontramos. Por otra parte, el rasgo de transferabilidad del modelo transpodidáctico hace posible que, como su nombre indica, se pueda transferir y adaptar a cualquier otro entorno educativo y cultural distinto del que hoy y aquí proponemos.

Según Moore (1980) una teoría educativa es fundamentalmente una teoría práctica y ésta no puede ser probada o refutada simplemente al hacer referencia al mundo empírico siendo necesario demostrar que sus conclusiones merecen ser puestas en práctica. Además hay que demostrar que sus conclusiones no se justifican a partir de los supuestos o bien que éstos son cuestionables. Por tanto, hay que tener en cuenta que dado que se hacen afirmaciones empíricas, este modelo puede ser contrastado con hechos también empíricos. Puesto que incluye juicios de valor, resulta que este modelo podría ser vulnerable a la argumentación filosófica $\mathrm{y}$, puesto que su argumentación es deductiva, también es cierto que puede ser sometida a una prueba de coherencia interna. Si nuestro modelo fallase en alguno de estos aspectos, hecho que no sucede hasta donde hemos podido alcanzar, entonces podría ser rechazado como guía de la práctica.

Otros aspectos que también pueden jugar un papel importante en la validación de cualquier teoría educativa son: a) el consenso social, que la comunidad apruebe una serie de prácticas educativas por considerarlas adecuadas y pertinentes; $b$ ) el consenso académico, según Kuhn (1961) una teoría se mantiene como válida mientras estos métodos sean los dominantes para una comunidad científica concreta que los acepta hasta que con el paso del tiempo las nuevas generaciones adopten un nuevo paradigma; c) también es importante obtener el consenso político, porque es en este marco donde se establecen a través de la legislación las reglas y métodos a seguir dentro de la práctica educativa. Por otra parte, d) es interesante tener en cuenta y obtener cierto consenso ideológico, en el que se incluyen los representantes del proceso educativo (padres, maestros y alumnos) para que den validez a las creencias de cómo debe de ser e implementarse la práctica educativa; e) el consenso económico, constituye un elemento clave porque los recursos económicos se facilitan siempre y cuando aquellos que los proporcionan estén de acuerdo con el tipo de práctica educativa que consideran válida o deseable. Por último, f) cierto consenso con respecto a una comunidad religiosa podría constituir un elemento de influencia que validase los supuestos morales de los métodos y prácticas educativas. 
Por tanto, y de acuerdo con las premisas de Moore (1980) que proporcionan el marco lógico conceptual para poder implementar si no una teoría educativa, al menos este modelo teórico-práctico, se ha establecido su estructura y cada uno de los supuestos en que debe de basarse. Además, estas premisas han permitido validar y establecer los criterios apuntando su pertinencia quedando defendida y justificada la validez del modelo teórico-práctico de la transpodidáctica textual.

\section{PROPUESTA CONCRETA: "Los trajes nuevos del emperador"}

Tal y como se ha citado anteriormente, nuestra propuesta se explica partiendo de cuatro puntos fundamentales. En primer lugar, una selección textual que concluye con la elección del texto inicial que, sin lugar a dudas, constituye una gran parte del éxito de la misma. En segundo lugar, explicaremos los pilares básicos y todos aquellos elementos necesarios para desarrollar el proceso de transformación y aprendizaje. En tercer lugar, el paso de la transformación del hipotexto en hipertexto, momento en el que se elabora la representación teatral por escrito $\mathrm{y}$, por último, su posterior representación dramática de forma oral. De este modo se practica el aprendizaje y uso de una lengua abarcando las cuatro destrezas en su totalidad.

\subsection{Selección y elección del texto de ficción literario inicial (TFL 1)}

El primer paso para iniciar la implementación del modelo transpodidáctico textual consiste en una selección de textos razonada. De acuerdo con el nivel educativo y teniendo en cuenta los distintos factores que influyen en el proceso de aprendizaje de una lengua conviene seleccionar un texto de ficción literario partiendo de diversas fuentes de información. Algunas de éstas podrían ser las que describimos a continuación, pero antes quisiéramos matizar que sería bueno dejar participar en la primera parte de la selección a las dos partes implicadas en el proceso: docente y discentes.

Por tanto, la selección textual puede hacerse teniendo en cuenta textos de ficción breves como las fábulas de Esopo, las de la Fontaine, las de Samaniego o las de Iriarte por poner algunos ejemplos. Las fuentes que abarcan la tradición oral contienen una gran cantidad de historias ficcionales que bien podrían servir de inspiración a la hora de abordar esta empresa. Las leyendas populares transmitidas de generación en generación constituyen también una fuente documental interesante que puede servir perfectamente de motor de arranque. Dentro de nuestra propia literatura española encontramos numerosos ejemplos de relatos breves que constituyen una valiosa fuente de información, pongamos por ejemplo los relatos breves y cuentos de escritores del siglo XX como Emilia Pardo Bazán o Wenceslao Fernández Flórez.

Entendiendo que un texto de ficción literario (TFL) puede resolverse de diferentes maneras desde el punto de vista de su tipología textual, todo aquel texto literario que contenga una historia de ficción o diégesis podría ser válido para iniciar un proceso transpodidáctico textual. Así pues, un poema épico o de otra 
índole, e incluso algunos cantos o rimas antiguas podrían también ser útiles para esta primera fase de selección textual. Por supuesto, los textos de ficción teatrales o textos de ficción fílmicos también tienen cabida y pueden ser el punto de partida en esta primera selección para acometer un proceso de esta índole.

Una vez hecha la primera selección damos paso a la elección de un único texto. Conviene centrarse en el TFL elegido habiendo tenido en cuenta las posibles versiones y traducciones existentes, pues gracias a la transmisión oral y textual una misma historia se va transformando de muchas y diversas maneras a lo largo del tiempo hasta llegar a nuestros días. En esas transformaciones intervienen aspectos históricos, sociales, políticos e incluso económicos que dotan a estas historias de veracidad $\mathrm{y}$, sobre todo, las actualizan. Un ejemplo claro de esta transmisión textual podemos encontrarlo en el cuento de Hans Christian Andersen (1805-1875) que hemos elegido para implementar este modelo: "The Emperor's New Clothes"1.

En primer lugar y en cuanto a la transmisión textual, a partir de la fecha de su publicación fueron muchas las versiones y traducciones posteriores en las que podríamos detenernos, pero es necesario hacer una retrospectiva y dar cuenta de un dato sorprendente: una de las obras cumbre de la narrativa en prosa de la Literatura Española del siglo XIV: El Conde Lucanor escrita por el infante Don Juan Manuel (1282-1348). Este libro constituye en su mayor parte un libro de exempla o cuentos moralizantes escrito en castellano antiguo entre los años 1330 y 1335, pero prestaremos especial atención al cuento XXXII que lleva por título: "Lo que sucedió a un rey con los burladores que hicieron el paño". Tras la lectura de este texto inmediatamente apreciamos que el cuento de Andersen sobre los trajes nuevos del emperador está inspirado, sin lugar a dudas, en este ejemplo escrito varios siglos antes en España aunque con las diferencias lógicas que proporcionan la distancia geográfica y la historia de cada lugar.

En segundo lugar, cabe destacar la condición de viajero incansable de Andersen y quizás como consecuencia de sus numerosos viajes al extranjero obtuviese las fuentes de inspiración para escribir historias y redactar sus cuentos. Andersen escribió también muchos libros de viajes en los casi nueve años que vivió fuera de Dinamarca y en uno de éstos titulado: I Spanien (ed. danesa de 1863) describió y narró hechos e historias relacionadas con sus viajes a Cartagena, Málaga, Toledo,

\footnotetext{
1 "The Emperor's New Clothes" está clasificado con el n ${ }^{\circ} 17$ en el registro de las obras literarias del Centro Hans Christian Andersen en la categoría de "cuento de hadas". Fue publicado por primera vez el 7 de abril de 1837 como parte de: Eventyr, fortalte for Børn. Første Samling. Tredie Hefte. ( $\left.\mathrm{N}^{\mathrm{o}} 303\right)$.

${ }^{2}$ Edición original en español (1330). Título original: Libro de los enxiemplos del Conde Lucanor et de Patronio. Adaptación María Jesús Lacarra Editorial Espasa Calpe. Madrid. Nueva edición (1999). Puede consultarse en:

http://www.cervantesvirtual.com/obra-visor/el-conde-lucanor--0/html/
} 
Burgos, Cádiz, Sevilla y otras ciudades españolas. Obviamente estos datos arrojan luz con respecto a la similitud entre las dos historias y, a nuestro juicio, dotan de rigidez a nuestra afirmación y parecen ser muy clarificadores.

Por último, para terminar con la selección textual, nuestra elección final está basada en el texto: "The Emperor's New Clothes" escrito en lengua inglesa y que fue traducido a la lengua inglesa por Jean Hersholt (1886-1956), un actor danés que emigró a los Estados Unidos y fue traductor incansable de las obras de Andersen en una excelente edición ${ }^{3}$. Esta edición y traducción en lengua inglesa es considerada por muchos como la traducción estándar en esa lengua y no por pocos como una de las mejores. Hersholt consiguió conectar profundamente con este escritor y no solo escribió bastantes artículos sobre él sino que también editó parte de su correspondencia en el año 1948.

\begin{tabular}{|c|l|l||}
\hline $\begin{array}{c}\text { TFL 1 } \\
\text { Selección }\end{array}$ & $\begin{array}{l}\text {-Señor conde -dijo Patronio-, tres pícaros fueron a palacio y dijeron al rey que eran } \\
\text { excelentes tejedores, y le contaron cómo su mayor habilidad era hacer un paño que } \\
\text { solo podían ver aquellos que eran hijos de quienes todos creían su padre, pero que } \\
\text { dicha tela nunca podría ser vista por quienes no fueran hijos de quien pasaba por } \\
\text { padre suyo. }\end{array}$ \\
\hline $\begin{array}{c}\text { TFL 1 } \\
\text { Elección }\end{array}$ & $\begin{array}{l}\text { Every day many strangers came to town, and among them one day came two } \\
\text { weave the most magnificent fabrics imaginable. Not only were their colors and } \\
\text { patterns uncommonly fine, but clothes made of this cloth had a wonderful way of } \\
\text { becoming invisible to anyone who was unfit for his office, or who was unusually } \\
\text { stupid. }\end{array}$ \\
\hline TFT 2 & Ejemplo 1 & $\begin{array}{l}\text { TAILOR 2.- You must trust us! We will weave the best suit you have } \\
\text { never seen! This suit is magical and only the wise people can see } \\
\text { them. }\end{array}$ \\
\hline TFT 2 & Ejemplo 2 & $\begin{array}{l}\text { EMPRESS.- Oh, it's the most lovely design that I've ever seen. } \\
\text { TAILOR 1.- It's so special that only the intelligent people can see it. }\end{array}$ \\
\hline
\end{tabular}

Figura 3. Selección, elección textual y dos ejemplos del resultado final.

${ }^{3}$ Jean Hersholt tradujo los cuentos e historias de Hans Christian Andersen en una excelente edición titulada: The Complete Andersen (seis volúmenes, New York 1949). El cuento citado puede consultarse en:

http://www.andersen.sdu.dk/vaerk/hersholt/TheEmperorsNewClothes_e.html (15/10/ 2011). 


\subsection{Proceso transpodidáctico textual $(Q)$}

Desde el mismo momento en que se elige el texto de ficción literario inicial tras la selección textual comienza el proceso transpodidáctico que tiene la finalidad de implementar aquellos cambios necesarios para la obtención de otro texto de ficción literario con la intención de alcanzar el aprendizaje y buen uso de una lengua extranjera. Para ello, es necesario tener en cuenta una serie de elementos que describiremos a continuación y que tienen su base principal tanto en los documentos marco de la Comunidad Europea ${ }^{4}$, como en el marco legislativo nacional en cuanto a la enseñanza y aprendizaje de lenguas en cualquiera de los niveles educativos de las enseñanzas regladas 5 .

De acuerdo con estos documentos, el primero de los factores a tener en cuenta consiste en establecer en qué medida la implementación de este modelo contribuye a la adquisición de alguna o todas las competencias básicas marcadas por el documento marco, a saber: 1- la competencia comunicativa del lenguaje, 2- la competencia matemática, 3- la competencia en el conocimiento y la interacción con el mundo físico, 4- la competencia en el tratamiento de la información y competencia digital, 5- la competencia social y ciudadana, 6- la competencia cultural y artística, 7- la competencia para aprender a aprender y, 8- la autonomía e iniciativa personal. En el caso que nos ocupa se promociona el desarrollo de la primera, la competencia comunicativa del lenguaje, con sus tres subcompetencias (la c. lingüística, la c. sociolingüística y cultural y la c. pragmática) como no podía ser de otro modo, pero también la competencia social y ciudadana y la competencia cultural y artística.

Con respecto a la competencia comunicativa del lenguaje, no cabe duda que el lenguaje, tanto si es en la lengua materna como en una lengua extranjera, constituye un instrumento tanto para la comunicación oral como para la comunicación escrita. Sirve también para representar, interpretar y comprender la realidad. Del mismo modo, nos ayuda a organizar el pensamiento y las emociones, implica además poder expresar pensamientos, emociones, vivencias y opiniones. Adquiriendo esta competencia somos capaces además de proporcionar coherencia y cohesión al discurso, de adoptar decisiones y también es posible disfrutar escuchando, leyendo o expresándose de forma oral y escrita.

Puesto que la metodología empleada para la implementación de este modelo promociona el trabajo en equipo, la toma de decisiones y la colaboración interactiva parece claro que también se contribuye al desarrollo de la competencia

4 Common European Framework of Reference for Languages: Learning, Teaching, Assessment. Strasbourg: Council of Europe, 2001.

${ }^{5}$ Real Decreto 1513/2006, de 7 de diciembre, por el que se establecen las enseñanzas comunes de la Educación Primaria. Documento BOE: 8 de diciembre de 2006. 
social y ciudadana. Además, el texto objeto de estudio por sus características y diégesis también proporciona elementos interesantes relacionados con esta competencia. Y, por último, la competencia cultural y artística también se desarrolla en la medida en que los participantes deben realizar una labor previa de inmersión cultural y literaria así como también deberán estar en posesión de conocimientos básicos en las artes escénicas para la representación final.

Puesto que el espacio de este capítulo es limitado no conviene extenderse en este punto pero, obviamente, los objetivos y contenidos a los que aluden los documentos oficiales, siempre dependiendo del nivel educativo al que vaya dirigida la propuesta, estarán encaminados a asegurar el desarrollo de las competencias básicas anteriormente señaladas. Dado que el ejemplo elegido ha sido implementado para un nivel de educación primaria damos paso a la relación de algunos de los objetivos planteados que deberán ser comprobados tras la implementación de este modelo teórico-práctico. Todos ellos parten de la base común extraída de los objetivos generales previstos para esta etapa en cuanto al aprendizaje de lenguas extranjeras.

Uno de estos objetivos consiste en conseguir que los sujetos sean capaces de expresarse e interactuar oralmente en situaciones sencillas y habituales utilizando tanto la comunicación verbal como la comunicación no verbal. En segundo lugar, deberán ser capaces también de escribir el texto de ficción literario final con la ayuda del texto de ficción literario inicial establecido previamente como modelo y punto de partida. Todo ello implica el ejercicio de una lectura de textos comprensiva de los cuales serán capaces de extraer información general y específica de acuerdo con una finalidad concreta. Otro objetivo consiste en valorar la lengua extranjera, y las lenguas en general, bien como medio de comunicación entre personas de procedencias y culturas diversas, o bien como una herramienta de aprendizaje siempre adoptando una actitud respetuosa y de cooperación.

Por último, es necesario presentar como otro objetivo a conseguir tras el proceso transpodidáctico textual, la posibilidad real que existe de mostrar una actitud receptiva y de confianza en la propia capacidad de aprendizaje y de uso de una lengua extranjera. Por ello, es importante resaltar que tanto la identificación de distintos aspectos relacionados con la fonética, el ritmo, la acentuación y la entonación, así como el uso de las estructuras lingüísticas y aspectos léxicos de la lengua extranjera usados como elementos básicos para la comunicación suponen un logro probado tras la implementación de este modelo.

En cuanto a los contenidos que establece el marco legislativo para esta etapa, sin entrar en profundidad, quisiéramos señalar que para la implantación de este modelo se tienen muy en cuenta los cuatro bloques de contenidos que marcan los documentos curriculares anteriormente citados atendiendo: en primer lugar, al uso y desarrollo de las destreza orales; en segundo lugar, al uso y desarrollo de las destrezas escritas; en tercer lugar, incluyendo en todo momento el uso y reflexión 
de la lengua en sí misma y, por último, todos aquellos aspectos relacionados con los elementos socio-culturales y la consciencia intercultural.

Con respecto a la metodología empleada para poner en práctica esta teoría conviene aclarar que, en términos muy generales, se enmarca dentro de lo que en Didáctica de la lengua Extranjera (DLE) se conoce como el enfoque comunicativo en la enseñanza de lenguas (Communicative Language Teaching) o para simplificar: el Enfoque Comunicativo (Communicative Approach) en el que se incluye la metodología más utilizada en cuanto a este tipo de aprendizajes (Richards \& Rodgers, 1986). El planteamiento de la enseñanza de lenguas a través del Enfoque Comunicativo constituye una tendencia metodológica nacida en Gran Bretaña en los años sesenta dentro del contexto de la enseñanza y aprendizaje de lenguas extranjeras que surgió con la finalidad de reaccionar contra los métodos empleados por los estructuralistas con anterioridad. Aunque, en un principio partía de las investigaciones realizadas por miembros de áreas de enseñanza y aprendizaje de lenguas extranjeras, pronto se incorporó también al área de enseñanza y aprendizaje de lenguas maternas por el hecho de que ambas áreas comparten y se complementan en muchos de sus supuestos teóricos.

Para el desarrollo y puesta en marcha de nuestra propuesta concreta hemos decidido centrar toda la atención en la metodología basada en el aprendizaje a través de proyectos (Project Based Learning) puesto que nos sirve para dotar de coherencia y consistencia a nuestro modelo teórico-práctico partiendo del enfoque comunicativo anteriormente citado. El aprendizaje a través de proyectos surgió hace más de cien años en Estados Unidos desde el mismo momento en que algunos educadores como John Dewey (1859-1952) decidieron centrar el proceso de aprendizaje directamente en el alumno y no en el profesor (Westbrook, 1991; Moss, 1998). Las raíces de esta metodología se encuentran en esta larga tradición profundamente asentada en el ámbito de la educación americana y no tanto en la europea. Se trata de un método de enseñanza y aprendizaje que involucra a los estudiantes en el aprendizaje de conocimientos y destrezas a través de un proceso estructurado siempre alrededor de un diseño de tareas complejo y programado. La duración de este tipo de proyectos puede oscilar entre una o dos semanas, si se trata de un proyecto breve, hasta un año completo en el que se suelen integrar proyectos interdisciplinares incluyendo a una comunidad educativa al completo tanto desde dentro como fuera del entorno académico/escolar.

Según algunos expertos en la elaboración e implementación de proyectos (Wrigley, 1998) las fases principales en las que se pueden dividir los mismos se resumen en los cinco apartados que enumeramos a continuación: selección, planificación, investigación, desarrollo y compartir resultados. Este tipo de proyectos incluyen estrategias de aprendizaje y comportamientos afectivos que presumen un efecto positivo en el aprendizaje de lenguas obligando al que los lleva a cabo tanto a asumir riegos, como a tomar decisiones, o a organizar y hacer uso de recursos tecnológicos, humanos y materiales (Van Duzer, 1994). 
Si comparamos la amplia trayectoria en el uso de esta metodología en los Estados Unidos con su introducción en contextos europeos, concretamente en España, parece claro que todavía queda mucho camino por recorrer, aunque cada vez son más los intentos de implementar el aprendizaje a través de proyectos (Trujillo, 2012; Ferrer, Algás y Martos, 2007) son muchos los obstáculos que se producen en el sistema educativo español, bien por desconocimiento, o por falta de coordinación, o de tiempo, etc..., y los que existen se deben a tentativas individuales o aisladas de profesorado comprometido con la innovación y la enseñanza de calidad.

Parece haber consenso general en la necesidad de adaptación de la educación a un mundo cambiante y, por ello, el aprendizaje a través de proyectos ofrece a aquellos que lo practican oportunidades reales de aprendizaje haciendo que éstos se involucren en el proceso educativo desde el principio hasta el final del mismo. Tanto la forma en que se producen estos aprendizajes, como los elementos implicados en los mismos, como los resultados finales obtenidos se desarrollan y materializan de un modo totalmente diferente al modo en que se resolvían por medio de los métodos tradicionales. Se ponen en marcha, pues, los grupos de trabajo de entre cinco y siete miembros que llevarán a cabo el proceso transpodidáctico transformando el texto inicial TFL 1 en otro final TFT 2 realizando todos sus aportaciones siempre con la perspectiva de realizar un aprendizaje colaborativo.

A continuación se marca una ruta de trabajo acordando y conviniendo que es necesario un total de cuatro sesiones de tres horas aproximadamente en las que el profesor actuará de guía para dirigir a los componentes de cada grupo con la finalidad de cumplir su objetivo final. La primera sesión se inicia con una introducción de conceptos relacionados con los textos literarios de ficción, planteándose la selección textual y todos aquellos aspectos relevantes para el desarrollo de la misma. Finalmente se lleva a cabo la elección del TFL1 que será el que se emplee como base para las sesiones posteriores y para la realización del proceso transpodidáctico. En esta sesión se combinan las clases magistrales o más tradicionales con la participación de todos los miembros de cada grupo desde el principio. La segunda sesión estará dedicada al proceso transpodidáctico en sí mismo teniendo en cuenta todas las indicaciones señaladas en los apartados anteriores. En la tercera sesión se ultiman los detalles de dicho proceso y se empieza a escribir el TFT 2 o producto final. Por último, la cuarta y última sesión se dedica al perfeccionamiento del TFT 2 en todos los aspectos didácticos y lingüísticos necesarios: gramática, ortografía, matices, valores, actualización, etc.

Sobre las actividades, materiales y recursos empleados en cada una de las sesiones no conviene extenderse dado el espacio restringido de este formato pero sí nos gustaría indicar que todos ellos están a disposición de todo aquel lector interesado en su implementación. En cuanto a los materiales utilizados es preciso apuntar la existencia de dos etapas claramente diferenciadas. Por una parte, el 
proceso llevado a cabo en el aula tanto con materiales auténticos y no auténticos y, por otra, todos los detalles y aspectos derivados de los materiales necesarios para la representación final.

Los criterios de evaluación atienden a las necesidades del proceso y siguen los indicadores del marco legislativo en el que se integran. Se parte de una evaluación continua y global que incluye aspectos derivados tanto de la auto-evaluación como del aprendizaje permanente tal y como recogen los documentos marco a los que hemos aludido anteriormente. Además, para evidenciar los resultados obtenidos convendría tener en cuenta diferentes agentes involucrados en todo el proceso educativo: los alumnos universitarios como futuros maestros, el profesorado de los centros educativos, el alumnado de Primaria al que va dirigida la propuesta, los padres y/o tutores, etc. En el supuesto caso de que se presentase esta propuesta ante un alumnado con necesidades especiales, o ante un grupo heterogéneo en el que fuese necesario prestar una especial atención a la diversidad proponemos ponerse en contacto con los especialistas habilitados par ello en el centro y coordinar desde el comienzo una propuesta ajustada a las necesidades del grupo.

Por último, no quisiéramos terminar este apartado sin mencionar la necesidad de resaltar dos conceptos importantes que aportan, en nuestra opinión, mayor calidad a este tipo de propuestas educativas. Gracias a la interdisciplinariedad a la que hemos aludido anteriormente se aborda el interesante concepto de la transversalidad por una parte y, por otra, es necesario tener en cuenta la posibilidad que ofrece el marco propuesto para desarrollar una educación en valores humanos y universales como: el orden, el respeto a los demás, la responsabilidad, la generosidad, etc., tan necesarios hoy en día para la convivencia entre las diferentes lenguas y culturas.

\subsection{Texto de ficción literario final (TFT 2)}

Para la elaboración del texto de ficción literario final se procede a la fase de escritura que resulta de la aplicación de los pasos a los que nos hemos referido en los apartados anteriores. Se trata pues de una obra teatral breve escrita en tres actos en lengua inglesa, o en la lengua que se determine, en la que se diferencian claramente tres elementos estructurales: la presentación, el nudo y el desenlace final coincidiendo éstos con cada uno de los tres actos. Cada acto estará dividido en dos o tres escenas en las que puede haber un narrador, si se estima oportuno, y los diálogos en los que intervienen los actantes. Durante el desarrollo de los acontecimientos, deberá determinarse claramente un punto climático preciso que provoque el desenlace final deseado. En el tratamiento práctico de la escritura teatral también convendría dar cuenta de las didascalias, de los espacios en el escenario, de los tiempos de la obra y de los actores.

Esta parte del proceso en la que fundamentalmente se promocionan las destrezas escritas se realiza en grupo siguiendo la metodología planteada en el apartado correspondiente. Tras una asamblea para concretar los aspectos generales de la propuesta, el profesor actúa como un guía o mediador que orienta y ayuda a 
tomar decisiones con respecto a posibles dudas de carácter lingüístico o gramatical que puedan surgir a medida que avanza la elaboración del texto escrito. El espacio más apropiado para esta fase es el aula de aprendizaje y las fuentes documentales y los materiales serán los propios del aula de lenguas (el cuento, diccionarios, libros de gramática, de consulta, etc.). A continuación, se muestran dos cuadros que servirán de ejemplo para justificar todo lo expuesto anteriormente. En ambos cuadros, que remiten al comienzo (figura 4) y el final de la historia (figura 5) respectivamente, se pueden observar claramente los cambios realizados del texto de ficción literario inicial al texto de ficción literario final resultante tras pasar por el filtro del proceso transpodidáctico textual.

\begin{tabular}{|c|l||}
\hline \hline TFL 1 & $\begin{array}{l}\text { Many years ago there was an Emperor so exceedingly fond of new clothes that he } \\
\text { spent all his money on being well dressed. He cared nothing about reviewing his } \\
\text { soldiers, going to the theatre, or going for a ride in his carriage, except to show off } \\
\text { his new clothes. He had a coat for every hour of the day, and instead of saying, as } \\
\text { one might, about any other ruler, "The King's in council," here they always said. } \\
\text { "The Emperor's in his dressing room." }\end{array}$ \\
\hline TFT 2 & $\begin{array}{l}\text { ACT 1, Scene I } \\
\text { STORY TELLER.- Once upon a time in a far, far away place there was an } \\
\text { Emperor who only was interested in fashion clothes. He didn't care about anything } \\
\text { else. One day, two swindlers arrived at the castle pretending to be tailors. }\end{array}$ \\
\hline
\end{tabular}

Figura 4. Comienzo de la historia: "The Emperor's New Clothes".

\begin{tabular}{||c|l||}
\hline TFL 1 & $\begin{array}{l}\text { "But he hasn't got anything on," a little child said. } \\
\text { "Did you ever hear such innocent prattle?" said its father. And one person } \\
\text { whispered to another what the child had said, "He hasn't anything on. A child says } \\
\text { he hasn't anything on." } \\
\text { "But he hasn't got anything on!" the whole town cried out at last. } \\
\text { The Emperor shivered, for he suspected they were right. But he thought, "This } \\
\text { procession has got to go on." So he walked more proudly than ever, as his } \\
\text { noblemen held high the train that wasn't there at all. }\end{array}$ \\
\hline TFT 2 & $\begin{array}{l}\text { ACT 3, Scene II } \\
\text { CHILD. - Mum look, the emperor is nude! } \\
\text { MOTHER. - Shhhh...! } \\
\text { CHILD. - Mum look, it's true. } \\
\text { emperor was nude so they started laughing. The emperor was embarrassed but he } \\
\text { decided to keep on walking normally, until getting home]. }\end{array}$ \\
\hline \hline
\end{tabular}

Figura 5. Final de la historia: “The Emperor's New Clothes”. 
Por tanto, es en este momento de desarrollo de las destrezas escritas donde se pueden observar, analizar y explicar todos aquellos aspectos relacionados con las competencias comunicativas de la lengua, especialmente: la competencia léxica, que atañe al conocimiento del vocabulario de una lengua y la capacidad para utilizarlo; la competencia gramatical, que se refiere a los recursos gramaticales de una lengua y la capacidad de utilizarlos; la competencia semántica, que alude a la conciencia y el control de la organización del significado y la competencia ortográfica, que presupone el conocimiento y la destreza en la percepción y la producción de los símbolos de que se componen los textos escritos. Mención aparte merecerían la subcompetencia sociolingüística y cultural y la subcompetencia pragmática de las que daremos cuenta más adelante.

\subsection{Puesta en escena y representación teatral (R)}

A la fase de elaboración del texto escrito le sigue un segundo momento claramente diferenciado en este apartado que aborda la puesta en escena y la representación teatral. En esta fase también se promocionan las destrezas productivas y se fomenta, sobre todo, el uso y aprendizaje de la lengua en su vertiente oral. Conviene apuntar que es conveniente estar al tanto de algunas nociones básicas y terminología teatral, así como tener en cuenta aspectos relacionados con la representación dramática y la puesta en escena para conseguir resultados satisfactorios. Sería conveniente también proponer unos ejercicios previos de respiración abdominal y de impostación de la voz, aunque sea a un nivel muy básico.

El papel del profesor se ve incrementado considerablemente en este momento hasta el punto de ejercer como director escénico. Las funciones del director escénico son muchas y variadas puesto que, además de todo el trabajo preliminar realizado en las etapas anteriores, es necesario ahora plantearse el reparto de personajes, los ensayos, el vestuario y la puesta en escena. Con respecto al reparto de los personajes, el director debe tener claro que todos los alumnos y alumnas del grupo tienen que participar. Cada cual lo hará en mayor o menor medida siempre teniendo en cuenta aspectos derivados de la personalidad, carácter y comportamiento de los alumnos.

Si bien es cierto que casi siempre existen personajes clave que suelen ser individuales, es interesante señalar que también hay personajes colectivos que pueden admitir un gran número de participantes. Por ejemplo, en: “The Emperor's New Clothes" puede haber un rey, uno o dos pajes, una reina, un primer ministro, etc., pero también hay un personaje colectivo que son los ciudadanos del reino presentes el día del desfile. En este personaje colectivo pueden participar un número indeterminado de actantes y además tendrían cabida aquellos de carácter más tímido o vergonzosos. Otra solución para que todos puedan hablar y sean protagonistas por un momento es la inclusión entre acto y acto, o entre escena y escena, del número de narradores que sea necesario (Casanova, 2012). Éstos irán 
saliendo a escena según se va contando la historia y nuestra recomendación es que lo hagan de uno en uno o, como máximo, de dos en dos dependiendo de las necesidades puntuales en cada caso.

En cuanto a los ensayos, el director escénico deberá facilitar un clima informal, de cohesión y empatía en el que se potencie el buen ánimo y el refuerzo positivo entre los participantes. Las críticas deberán ser constructivas dejando espacio a la discusión y nunca a la imposición. Tampoco conviene interrumpir, ni adquirir protagonismo a no ser que surjan cuestiones concretas y, por último, tampoco sería conveniente sermonear, ni presionar por resultados (Kerman, 1961).

Dependiendo del tiempo y sesiones fijadas en el plan de trabajo convendría diferenciar, al menos, tres momentos: un primer momento de lectura oral del texto teatral con los personajes con la finalidad de mejorar todos aquellos aspectos derivados de la competencia fonológica, que se ocupa del conocimiento y la destreza en la percepción y la producción de las unidades de sonido (fonemas), de los rasgos distintivos de la lengua: sonoridad, nasalidad, oclusión, labialidad, etc. y también otros aspectos como: el tono de voz, la acentuación, la pronunciación, la entonación y el ritmo, etc. A continuación se muestran otros dos ejemplos que servirán de explicación. En ambos se recomienda que el que desempeñe el papel de emperador emplee un tono grave, un ritmo pausado y una pronunciación impecable puesto que se supone que su cargo evidencia una buena posición social. Asimismo realizará una entonación adecuada de acuerdo con las premisas de la lengua inglesa acentuando aquellos términos (marcados en negrita) que sirvan para enfatizar su disgusto y desaprobación contra el sastre real.

\begin{tabular}{|c|c|}
\hline $\begin{array}{l}\text { TFT 2/ R } \\
\text { Ejemplo } 1\end{array}$ & $\begin{array}{l}\text { Act I, scene I } \\
\text { EMPEROR.- Oh my God!, It's horrible! I'm tired about your designs, I want } \\
\text { something different. You are dismissed! }\end{array}$ \\
\hline $\begin{array}{l}\text { TFT 2/ R } \\
\text { Ejemplo } 2\end{array}$ & $\begin{array}{l}\text { Act I, scene II } \\
\text { EMPEROR.- Good morning, my lady! I had to dismiss the royal tailor because } \\
\text { I'm tired about his designs. I need a new tailor now! }\end{array}$ \\
\hline
\end{tabular}

Figura 6. Dos ejemplos de la representación oral enfatizando aspectos discursivos.

Pero dentro de las competencias comunicativas de la lengua no solo se desarrolla la adquisición de todas las competencias lingüísticas anteriormente citadas, sino también la competencia sociolingüística y cultural y la competencia pragmática. La competencia sociolingüística y cultural comprende el conocimiento y las destrezas necesarias para abordar la dimensión social del uso de la lengua y la competencia pragmática se refiere al conocimiento y estudio del lenguaje en relación con la comunicación en sí misma. Por ello, debe existir un segundo 
momento, en el que se realizan los ensayos por actos y escenas en el que, además de los anteriores, se han de tener en cuenta los aspectos relacionados con la kinesia (estudio del lenguaje corporal) y la proxemia (disciplina que aborda la relación entre el espacio personal y las características de cada individuo).

Por último, tendrán lugar los ensayos generales en los que se ultiman detalles técnicos, de vestuario y caracterización, que culminan con un ensayo general final previo al día fijado para la representación oficial. Tanto el vestuario y la caracterización como la puesta en escena final dependerán, en gran medida, de las posibilidades de los agentes participantes y de la capacidad y logística disponible de los centros o espacios en los que se pretenda implementar esta propuesta. Pero la experiencia ha demostrado que con un poco de imaginación, dedicación y esfuerzo es posible llevar a buen término, en un porcentaje muy elevado de éxito, la representación teatral resultante del proceso transpodidáctico textual implementado para la enseñanza y aprendizaje de lenguas.

\section{CONCLUSIÓN}

En el apartado de conclusiones conviene aclarar que, si bien algunas de las ideas que se recogen en este artículo ya han sido desarrolladas e implementadas por otros investigadores y docentes en el área objeto de estudio, hasta ahora no habían sido investigadas y planteadas desde un punto de vista científico y riguroso. Tampoco existía hasta ahora un modelo teórico-práctico que materializase de forma contundente los esfuerzos que muchos profesores de los distintos niveles educativos vienen realizando sistemáticamente en cuanto a la implementación de textos de ficción en las aulas de enseñanza y aprendizaje de lenguas propias y extranjeras. El hecho de que hasta la fecha no existiese un método riguroso y ampliamente desarrollado que validase las propuestas realizadas por muchos docentes en cuanto al aprendizaje de lenguas a través de textos de ficción ha impedido, en nuestra opinión, que se valoren debidamente los esfuerzos realizados en muchos centros educativos a lo largo y ancho de la geografía española.

Por otra parte, con el modelo transpodidáctico textual hemos acuñado en este artículo un nuevo término que solo el tiempo dirá si es apropiado o no dependiendo del uso que los futuros investigadores y docentes puedan hacer del mismo. Nuestro modelo desarrollado en cuatro fases facilita la comprensión y fácil implementación del mismo en distintos y variados ejemplos tal y como apuntábamos en apartados anteriores. Además, también hemos comprobado que se podría llevar a cabo en los distintos niveles educativos del sistema español con las consiguientes modificaciones que cada nivel ofrece en cuanto a competencias, objetivos y contenidos de etapa.

Al facilitar un ejemplo concreto en el que se explican y desarrollan cada una de las etapas por las que transcurre dicho modelo hemos querido probar su validez empírica, la veracidad y la utilidad del mismo. Además, se ha contribuido al desarrollo de las ocho competencias básicas, reforzando el uso y adquisición de las 
cuatro destrezas de la lengua y siempre teniendo en cuenta los bloques de contenidos que marca el currículo nacional. Por último, quisiéramos apuntar que la implementación del modelo teórico-práctico de la transpodidáctica textual en el ámbito de la enseñanza y aprendizaje de lenguas facilita el aprendizaje significativo, contribuye a que éste sea colaborativo y favorece la revisión, la evaluación permanente así como la auto-evaluación, elementos todos ellos importantes en la evolución de cualquier proceso educativo.

Tal vez, en un futuro no muy lejano, el modelo presentado en esta propuesta sea tenido en cuenta por los nuevos investigadores y refrendado por los docentes de los centros educativos dotando de validez científica las prácticas docentes. Prácticas docentes que éstos han venido poniendo en marcha durante muchos años tal vez de forma aislada y anónima y que, en la mayoría de los casos, se han quedado en una mera anécdota reducida a una representación teatral para padres al finalizar el curso escolar, eso sí, a ser posible en una lengua extranjera. El gran esfuerzo realizado por todos estos docentes anónimos para la consecución de un proyecto de esta magnitud y repercusión en la enseñanza-aprendizaje de lenguas merecía ser recompensado y por ello hemos querido dejar constancia a través de este artículo.

\section{BIBLIOGRAFÍA}

BLACK, James A. and CHAMPION, Dean J. (1976): Methods and Issues in social research, New York, Wiley.

CHEVALLARD, Yves (1985): La transposition didactique; du savoir savant au savoir enseigné, Paris, La Pensée Sauvage.

CASANOVA FUENTES, Andrea (2012): "Estrategias metodológicas para la enseñanza del inglés como lengua extranjera: La dramatización de textos en el aula". TFM del Máster Oficial en Innovación, Orientación y Evaluación Educativa Facultad Ciencias de la Educación. UDC. Dirigido por: Pilar Couto Cantero y Mercedes González Sanmamed.

COUTO CANTERO, Pilar (1999): "Teoría de la transposición cinematográfica. En defensa de los nuevos soportes. Discurso literario vs. discurso fílmico", en Cien Años de Cine. Historia, Teoría y Análisis del Texto Fílmico, CASTRO DE PAZ, José L., COUTO CANTERO, Pilar y PAZ GAGO, José Ma (eds), Madrid, Visor, 317-324.

DÍAZ CORRALEJO, Joaquín (2002): "Reflexiones sobre la Didáctica de la enseñanza/aprendizaje de la Lengua y la Literatura" en ARBOR CLXXIII, 681, septiembre, 129-152.

FERRER, Carolina, ALGÁS, Pilar, y MARTOS, Juan M. (2007): "Valoramos el trabajo por proyectos", Aula de Innovación Educativa, 166, 71-75.

HERNÁNDEZ SAMPIERI, Roberto, FERNÁNDEZ COLLADO, Carlos y BAPTISTA LUCIO, Pilar (1998): Metodología de la investigación (2a ed.), México D. F., McGraw-Hill. 
GÓMEZ MENDOZA, Miguel A. (2005): "La transposición didáctica: historia de un concepto" en Revista Latinoamericana de Estudios Educativos, Volumen 1, Julio - Diciembre, 83-115.

HERRERO FIGUEROA, Araceli (2000): "Los estudios de Literatura Infantil en la Diplomatura de Maestro. Un ejemplo de transposición didáctica”, Literatura Infantil y Juvenil. Tendencias actuales en investigación, Servicio de Publicaciones de la Universidad de Vigo, 59-67.

KERLINGER, Fred N. (1975): Investigación del comportamiento: técnicas y metodología, México, Nueva Editorial Interamericana.

KERMAN, Gertrude L. (1961): Plays and Creative Ways with Children, New York, Harvey House.

KUHN, Thomas S. (1962): The Structure of Scientific Revolutions, Chicago, University of Chicago Press.

KUHN, Thomas S. (1979): La estructura de las revoluciones cientificas, México, F.C.E.

MOORE, Terence W. (1974): Introducción a la teoría de la educación, Madrid, Alianza Universidad, 1980.

MOSS, Donna (1998): Project-based learning and assessment: A resource manual for teachers, Arlington, VA, The Arlington Education and Employment Program (REEP).

RICHARDS, Jack and RODGERS, Theodore (1986): Approaches and Methods in Language Teaching, Cambridge, Cambridge University Press.

TRUJILLO SÁEZ, Fernando (2012): "Enseñanza basada en proyectos: una propuesta eficaz para el aprendizaje y el desarrollo de las competencias básicas", Eufonía - Didáctica de la Educación Musical, 2012, num. 55, 7-15.

VAN DUZER, Carol (1994): Report to the adult education network. Arlington, VA, Arlington Education and Employment Program (REEP).

WESTBROOK, Robert (1991): John Dewey and American democracy, Ithaca, Cornell Universiry Press.

WRIGLEY, Heide S. (1998): "Knowledge in action: The promise of project-based learning", Focus on Basics, 2 (D), 13-18.

\section{PÁGINAS WEB}

Libro de los enxiemplos del Conde Lucanor et de Patronio. [en línea]. Adaptación María Jesús Lacarra Editorial Espasa Calpe. Madrid. Nueva edición (1999). Ref. de 16 de septiembre de 2011). Disponible en web:

http://www.cervantesvirtual.com/obra-visor/el-conde-lucanor--0/html/

HERSHOLT, Jean: "The emperor's new clothes" [en línea]. (Traducción en ingles). (Ref. de 15 de octubre de 2011). Disponible en web:

http://www.andersen.sdu.dk/vaerk/hersholt/TheEmperorsNewClothes_e.html 


\section{OBRAS DE REFERENCIA}

ADAM, Jean Michel (1990): Elements de linguistique textuelle: théorie et pratique de l'analyse textuelle, Paris, Mardaga.

AREIZAGA ORUBE, Elisabet (2000): "El enfoque comunicativo. Propuestas didácticas" en Ruiz Bikandi, U. (ed.): Didáctica de la segunda lengua en educación infantil y primaria, Madrid, Síntesis, 137-162.

ATHIEMOOLAM, L. (2004): Drama-in-education and its effectiveness in English Second/Foreign Language classes. (Ref. de 22 de octubre de 2013). Disponible en web: http://goo.gl/U3wLW.

ATIENZA, José Luis (1992): "De la teoría y de la práctica en la Didáctica de las Lenguas Extranjeras" en Las didácticas específicas en la formación del profesorado. (Vol. 1). MONTERO MESA, L. y VEZ JEREMÍAS, J. M. (eds.): Santiago de Compostela, Tórculo, 213-232.

BELLO, Pilar et al. (1990): Didáctica de las segundas lenguas: Estrategias y recursos básicos, Madrid, Santillana.

BESTARD MONROIG, Juan y PÉREZ MARTÍN, Ma Concepción (1992): La Didáctica de la Lengua Inglesa. Fundamentos lingüísticos y metodológicos, Madrid, Síntesis.

BOLTON, G. (1984): Drama as education: An argument for placing drama at the centre of the curriculum, Essex, England, Longman.

BRONCKART, Jean Paul y PLAZAOLA GIGER, Itziar (1996): "Théorie des actes de langage et enseignement de la L2. Un exemple de transposition didactique", Diálogos Hispánicos, 18, 13-35.

BROWN, Gillian (1996): Performance and Competence in Second Language Acquisition, (edited by Gillian Brown, Kirsten Malmkjaer and John Williams), New York, Cambridge University Press.

CANTERO, Francisco José; MENDOZA, Antonio y ROMEA, Celia (Eds.)(1997): Didáctica de la Lengua y la Literatura para una sociedad plurilingüe del siglo $X X I$, Barcelona, SEDLL-Publicaciones de la Universidad de Barcelona.

COUTO CANTERO, Pilar (2001): "Literatura y cine. Espacio literario y espacio fílmico en El malvado Carabel (1931/1955)" en Lecturas: imágenes. (Becerra, C., Candelas, M. A., Chas, A., Fariña, M. J. y Suárez, B. Eds.), Vigo, Servicio de Publicaciones de la Universidad de Vigo, 361-374.

COUTO-CANTERO, Pilar \& FERNÁNDEZ MACEIRAS, Tanya (2011): "Implementing and Supporting Drama in the EFL Early Childhood Classroom through Storybooks - A Classroom Action-Research in a Bilingual School in Spain" in Proceedings of the 3rd Paris International Conference on Education, Economy and Society, TCHIBOZO, G., Ed. Strasbourg (France), Analytrics 155-162. En http://analytrics.org/Documents/Actes\%20\%20Proceedings\%202011.pdf 30 de junio de 2011.

COUTO-CANTERO, Pilar (2011): "La promoción del multiculturalismo a través del cuento infantil" en Actas del XII Congreso Internacional de Literatura 
Española Contemporánea. El papel de la literatura, el cine y la prensa (TV/ Inernet/MAV) en la configuración y promoción de criterios, valores y actitudes sociales, LÓPEZ MARTÍNEZ, D. (Ed.), Andavira, 107-119.

COUTO-CANTERO, Pilar (2011): "Aproximación a la DLL en España" en La investigación en Didáctica de la lengua y la Literatura: situación actual y perspectivas de futuro, NÚÑ̃Z DELGADO, P. y J. RIENDA (Coords.) 19772002.

COUTO-CANTERO, Pilar (2011), "Teaching and Learning EFL through PBL", Sociology Study, September 2011, Volume 1, Number 4, 272-281.

DODSON, Sarah. L. (2000): FAQs: Learning languages through drama. Texas Papers in Foreign Language Education, 5(1), 129-141.

EL-NADY, M. (2000): Drama as a teaching technique in the Second Language classroom, Dialog on Language Instruction, 14(1, 2), 41-48.

FRIED-BOOTH, Diana (1997): Project work. (8th Ed.) Oxford, Oxford University Press.

FURMAN, Lou (2000): "In Support of Drama in Early Childhood Education, Again", Early Childhood Education Journal, Vol. 27, No 3, 2000.

HEARN, Izabella y GARCÉS Antonio (coords.) (2003): Didáctica del Inglés para Primaria, Madrid, Pearson Educación.

LEVINSON, S. C. (1980): "Speech act theory: the state of the art" en Language Teaching \& Linguistics: Abstracts (A survey article), 13, 1. 5-24.

LIU, J. (2002): Process drama in second-and foreign-language classrooms. In Gerd Bräuer (Ed.), Body and language. Intercultural learning through drama (pp. 5170), Westport, Connecticut \& London: Ablex Publishing.

MALEY, A. \& DUFF, A. (1978): Drama Techniques in Language Learning. Cambridge, Cambridge University Press.

MARTÍN VEGAS, Rosa A. (2009): Manual de didáctica de la lengua y la literatura, Madrid, Síntesis.

MENDOZA FILLOLA, Antonio y CANTERO SERENA, José (2003): "Didáctica de la Lengua y de la Literatura: aspectos epistemológicos" en Didáctica de la Lengua y la Literatura para Primaria, MENDOZA FILLOLA, A. (Coord.): Madrid, Pearson Educación, 3-32.

MENDOZA FILlOLA, Antonio (Coord.) (1998): Conceptos Clave en Didáctica de la Lengua y la Literatura, Barcelona, SEDLL - ICE- Horsori.

NUSSBAUM, Lucy y BERNAUS, Mercedes (2001): Didáctica de las lenguas extranjeras en la Educación Secundaria Obligatoria, Madrid, Síntesis.

PÉREZ VALVERDE, Cristina (2002): "Theatre in Education (TIE) in the Context of Educational Drama", Lenguaje y Textos, 20, 7-20.

RODRÍGUEZ LÓPEZ-VÁZQUEZ, Alfredo (Ed.)(1993): Simposio "O teatro e o seu ensino", La Coruña, Servicio de Publicaciones de la UDC.

RODRÍGUEZ LÓPEZ-VÁZQUEZ, Alfredo (1997): "Elementos didácticos del teatro" en Didáctica de la Lengua y la Literatura para una sociedad plurilingüe 
del siglo XXI, CANTERO, F. J., MENDOZA, A. y ROMEA C. (Eds.): Barcelona, Publicaciones de la Universidad de Barcelona, 401-404.

ROMERA CASTILlO, José (1978): Didáctica de la Lengua y la Literatura, Método y Práctica, Madrid, Playor, (7ª ed.)1988.

RUIZ BIKANDI, Uri (ed.) (2000): Didáctica de la segunda lengua en educación infantil y primaria, Madrid, Síntesis.

STERN, H.H. (David) (1984): Fundamental concepts of language teaching, Oxford University Press.

Tejerina, Isabel (1994): Dramatización y teatro infantil. Dimensiones psicopedagógicas y expresivas, Madrid: Siglo XXI.

- - - - (2000): "La literatura dramática infantil. Luces y sombras", ADE Teatro. Revista de la Asociación de Directores de Escena de España, 80; pp. 102-107.

VEZ JEREMÍAS, José M. y MONTERO MESA, Luis (1993): Las Didácticas Específicas en la formación del profesorado, (2 vols.), Santiago, Tórculo. 